\title{
EDITORIAL
}

\section{Directions to Contributors: stylistic matters}

All publications, from the tabloid newspapers to the proceedings of the most distinguished publishing house, have 'style guides' that form the basis of guidance to contributors and, more especially, the conventions used by the technical editors marking the typescripts for the printer. These style guides for most scientific journals, including the British Journal of Nutrition, consist of two parts; the first is concerned with scientific issues and deals with issues of nomenclature, units and the general structure of the papers published, and the second with linguistic stylistic matters. As with most journals, the current style guides have evolved over time in a rather ad hoc fashion, and the professional staff of the British Journal of Nutrition felt that it was time to look at the style guide as a whole to see whether we were content with the existing series of rules.

Some of the results of these discussions will appear in the printed Directions to Contributors but most of them will be used in technical editing, and I thought that it would be useful to share some of the spirit of these discussions with you and to explain the rationale for some of the changes. The scientific stylistic issues are reasonably straightforward. They are essential to ensure that scientific accuracy is maintained and that all readers will be able to identify the organisms mentioned in the Journal, that the systematic names of reagents and other constituents are named according to the agreed international conventions and the strengths of reagents are given unambiguously. In general we use the SI conventions, although occasionally the conventions cause difficulties with some clinical authors who have grown familiar with other forms of expression. I think that following the SI conventions rigidly is unnecessary provided that readers are not confused by the use of non-SI units where these units are in regular day-to-day usage.

The layout of tables and standards for illustrations are stylistic issues that relate to the appearance of the Journal and have been chosen as formats that are consistent with the design of the Journal. There are also some technical features which affect the processes involved in formatting the tables by the printer. Any tables that do not conform to the 'Journal Style' require extensive work by the technical editors, and this increases the probability of introducing error or departing from what the authors intended. We are reluctant to send papers back because the tables do not have the correct layout, but it is in the authors' interests to prepare tables in a form that conforms to our style.

The stylistic guides for figures are essential to ensure that the figure will reproduce accurately in print. Unfortunately some computers produce figures that are not adequate for the press, and I would urge all authors to make sure their figures are of adequate technical quality to reproduce properly in print.

We also discussed some conventions that have developed over the years; there were for example inconsistencies in the use of hyphenation and the use of italics for Latin terms. We hope to eliminate the inconsistencies that have caused annoyance to some authors and will use the Oxford English Dictionary as our guide in spelling and hyphenation.

The Journal has had a rigorous policy of not permitting any abbreviations without first citing them in full; we think that this is out of tune with the times and it is unnecessary to write out in full substances such as DNA and many other well-recognized and widely used abbreviations in the literature such as HPLC and GLC. We will, therefore, be relaxing this 
convention and will propose a list of abbreviations that do not need to be defined. We also think that the use of symbols for the chemical elements should be permitted, although I must say that I nearly had second thoughts at a meeting recently when a questioner asked why the ratio of phosphorus to sulphur should influence coronary heart disease!!

Professor Kon, the first editor of the Journal, aided by an Editorial Board that included A. L. Bacharach, set very high standards for the English construction of the papers published in the Journal, and many older authors will have bitter memories of receiving a manuscript which had been substantially rewritten by the editors. I found some of the stylistic rules rather rigid.

I am equally committed to scientific accuracy and clarity and I recognize that all the papers in the Journal need to be clear to readers who do not have English as their first language. Nevertheless, I also think that absolute conformity to a series of rigid conventions can produce an anonymous, bland and impersonal style which I find unattractive to read. I also believe that the English language is a living one that is evolving continuously, not only in the more literary sense but also as a scientific language.

Over the years successive Editors have adopted more relaxed stylistic standards, and I believe that the correct criteria for acceptance are that the paper should read well and be scientifically accurate. I do not see any conflict between good science and literary quality. I believe that as scientists we should be aiming to make the scientific literature worthy of its name (Maddox, 1990).

Such an approach will benefit the development of the nutritional sciences because wellwritten papers will be read rather than merely consulted, and if an element of personal style is evident readers may gain a better idea of how the science has really developed.

Maddox, J. (1990). Does the literature deserve the name? Nature 348, 191.

D. A. T. SouthGate 